\title{
Quantifying slow evolutionary dynamics in RNA fitness landscapes
}

\author{
Sulc, P ; Wagner, A ; Martin, O C
}

\begin{abstract}
We re-examine the evolutionary dynamics of RNA secondary structures under directional selection towards an optimum RNA structure. We find that the punctuated equilibria lead to a very slow approach to the optimum, following on average an inverse power of the evolutionary time. In addition, our study of the trajectories shows that the out-of-equilibrium effects due to the evolutionary process are very weak. In particular, the distribution of genotypes is close to that arising during equilibrium stabilizing selection. As a consequence, the evolutionary dynamics leave almost no measurable out-ofequilibrium trace, only the transition genotypes (close to the border between different periods of stasis) have atypical mutational properties.
\end{abstract}

DOI: https://doi.org/10.1142/S0219720010005075

Posted at the Zurich Open Repository and Archive, University of Zurich

ZORA URL: https://doi.org/10.5167/uzh-43095

Journal Article

Accepted Version

Originally published at:

Sulc, P; Wagner, A; Martin, O C (2010). Quantifying slow evolutionary dynamics in RNA fitness landscapes. Journal of Bioinformatics and Computational Biology, 8(6):1027-1040.

DOI: https://doi.org/10.1142/S0219720010005075 
Journal of Bioinformatics and Computational Biology

(C) Imperial College Press

\title{
QUANTIFYING SLOW EVOLUTIONARY DYNAMICS IN RNA FITNESS LANDSCAPES
}

\author{
P. ŠULC \\ Univ Paris-Sud, UMR8626, LPTMS, Orsay, F-91405; \\ CNRS, Orsay, F-91405, France \\ petr.sulc@polytechnique.edu \\ A. WAGNER \\ University of Zürich, Department of Biochemistry \\ Winterthurerstrasse 190 \\ CH-8057 Zürich, Switzerland \\ aw@bioc.uzh.ch \\ The Santa Fe Institute \\ 1399 Hyde Park Road, Santa Fe, NM 87501, USA \\ O. C. MARTIN \\ Univ Paris-Sud, UMR8626, LPTMS, Orsay, F-91405; \\ CNRS, Orsay, F-91405, France \\ olivier.martin@u-psud.fr \\ UMR de Génétique Végétale, INRA - Univ Paris-Sud - CNRS - AgroParisTech, \\ Ferme du Moulon, F-91190, Gif-sur-Yvette, France
}

\begin{abstract}
We re-examine the evolutionary dynamics of RNA secondary structures under directional selection towards an optimum RNA structure. We find that the punctuated equilibria lead to a very slow approach to the optimum, following on average an inverse power of the evolutionary time. In addition, our study of the trajectories shows that the out-of-equilibrium effects due to the evolutionary process are very weak. In particular, the distribution of genotypes is close to that arising during equilibrium stabilizing selection. As a consequence, the evolutionary dynamics leave almost no measurable outof-equilibrium trace, only the transition genotypes (close to the border between different periods of stasis) have atypical mutational properties.
\end{abstract}

\section{Introduction}

In many realistic systems, dynamics undergo a pronounced slow-down, a feature characteristic of "complex" landscapes ${ }^{1}$. Such phenomena arise for instance in physical systems (thermal relaxation ${ }^{2}$ ), in optimisation problems (diminishing returns on search efforts ${ }^{3}$ ), and in evolutionary dynamics (punctuated equilibria, i.e., long periods of stasis in the evolutionary record ${ }^{4}$ ). In this work, inspired by 
the theoretical framework of statistical physics for glassy systems, we reconsider a simple model of evolutionary dynamics, namely RNA secondary structure evolution $5,6,7,8$. We find: (i) slow evolutionary dynamics, whereby the time to find an advantageous phenotypic change has a distribution with a fat (power-law) tail; (ii) non-self-averaging behavior, i.e., even for long RNA molecules, directional selection for some targets will lead to significantly slower dynamics than for other targets; (iii) only weak out-of-equilibrium effects: the genotypes visited by an evolutionary trajectory are quite similar to those arising in equilibrium under stabilizing selection, so except near the ends of periods of stasis, the genotypes produced during an evolutionary trajectory do not have anomalously high or low mutational robustness.

The paper is organised as follows. In Sect. 2 we introduce the evolutionary model. In Sect. 3 we exhibit the non-exponential nature of the relaxation process; empirically, relaxation seems to follow an inverse power law. We also show that the relaxation curves remain sensitive to the target used for directional selection even in the limit of very long molecules. Finally in Sect. 4 we give evidence that at nearly all times the evolutionary trajectory is in quasi-equilibrium; more precisely, the "innovative" genotypes produced by a transition to a new period of stasis are only a bit different from random genotypes as measured by the phenotypic effects of mutations; furthermore, during the periods of stasis, the initial genotype seems to be quickly forgotten, so no significant trace of innovation seems to be maintained. Concluding remarks are given in the final section.

\section{The RNA evolutionary model}

\subsection{RNA structure and its fitness landscape}

For the purpose of this study, RNA molecules will be thought of as chains or strings of $L$ nucleotides, taken from an alphabet of four possible nucleotides (A, C, G and $\mathrm{U})$. Chemically, the bases can pair via hydrogen bonds. In addition to Watson-Crick pairings (A-U and G-C), the U-G pairing is also possible, though it is weaker. The pairings between bases give rise to an RNA secondary structure, as illustrated in Fig.1. Apart from this graphical representation, the secondary structure of an RNA molecule can be specified by the more convenient dot-bracket notation where a nonpaired base of the sequence is denoted by a dot "." and a paired base is denoted by a left or right parenthesis. This representation allows one to reconstruct the pairings as long as the secondary structure is "planar" ${ }^{9}$. (Planarity means that if the $L$ bases are positioned on a line and the pairings are represented by arcs between the bases, these arcs can be drawn in the plane without any crossings.) An RNA molecule will spontaneously (i.e., via thermodynamic forces only) fold into the structures with lowest free energies. To simulate this folding in silico, we use the "Vienna RNA package" 10 to find, for any given sequence, the pairings (secondary structure) which lead to the minimum free energy. In effect, this procedure produces a map from sequences (genotypes) to secondary structures (phenotypes) ${ }^{11,7,5,6,8}$. The CPU time needed to determine a minimum free energy structure is $O\left(L^{3}\right)$ for 


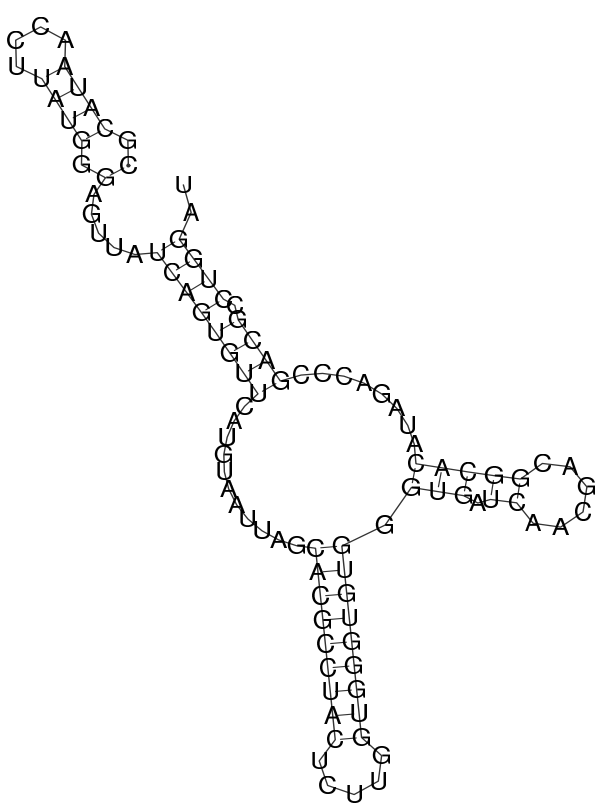

Fig. 1. Example of a secondary structure of an RNA molecule. Several typical features characteristic of RNA secondary structures are shown. They include hairpin loops at the periphery and a multiloop at the center. The structure shown is represented by $(.((((\ldots)))).) \ldots \ldots .(((((((\ldots \ldots \ldots \ldots . .((((((((\ldots \ldots))))))))) \cdot(((.((\ldots \ldots)))).) \ldots \ldots \ldots .)))))))$.$) .. in dot-bracket no-$ tation.

a chain of $L$ bases.

All genotypes that have the same phenotype form a neutral network ${ }^{11}$. More precisely, given a genotype to phenotype map, the neutral network associated with the phenotype $P$ is a graph whose nodes are all the genotypes having that phenotype; the edges of that graph connect genotypes if and only if they are nearest neighbors. It is thus necessary to introduce a notion of neighborhood in genotype space. For the present work, two RNA genotypes (sequences) will be considered as nearest neighbors if and only if they differ by a single base. Other choices would have been possible ${ }^{11}$ such as allowing a difference of two bases, meaning that two successive point mutations would transform one genotype to one of its neighbors.

One may also want to consider multiple phenotypes, leading one to also define a distance $d_{p}$ between two phenotypes. In our case, we take the distance between two RNA secondary structures $P_{1}$ and $P_{2}$ to be $1 / L$ times the Hamming distance between their dot-bracket string representations. Again, other choices of phenotypic distance functions have been used in the literature ${ }^{11,13}$ often interchangeably, but we use only the Hamming distance one for its simplicity. Given the neighborhood definitions both for genotypes and phenotypes, one can introduce a fitness landscape ${ }^{14}$, where genotype and phenotype spaces both have a distance metric, and where 
each phenotype can be assigned a "fitness". We take the fitness of a phenotype to be a monotonically decreasing function of its distance to some "target" phenotype $P_{t}$. For specificity, the reader can think of a decreasing exponential of distance, but as will be clear later on, the choice of function makes no difference. Finally, we will also extend the notion of a neutral network to that of a neutral ensemble: we call neutral ensemble the union of all neutral networks whose corresponding phenotype has a given fitness, regardless of the specific phenotype. This definition reflects that fact that secondary structures are relevant in the evolutionary search mainly via their distance to $P_{t}$. RNA sequences with their associated fitness form a fitness landscape with hills, valleys and passes (saddles). Analogous landscapes also arise in other systems; for example spin glasses ${ }^{15}$ are physical materials in which energy is often identified with fitness; the landscape's ruggedness and many valley structure are important for the associated dynamical properties.

\subsection{Dynamics under directional selection}

We take the unit of time to be the expected waiting time $\Delta t$ between two mutations. Thus if $\mu$ is the mutation rate per RNA molecule and per generation, our unit of time is $\Delta t=1 / \mu$. For our evolutionary dynamics, we simulate the process of RNA evolution towards a target structure by allowing a genotype to change by a point mutation at each unit of time. The directional selection associated with this evolutionary search then proceeds as follows. At each step, the genotype is mutated at one base taken at random; the corresponding phenotype (secondary structure for that genotype) is determined, and selection is applied: if the distance to the target structure has increased, the mutation is refused and the previous genotype is reinstated, otherwise the new genotype is accepted. This process is called "blind ant" dynamics ${ }^{17}$ but it also is referred to as an "adaptive walk" ${ }^{18}$. It can also be thought of as using Metropolis Monte Carlo ${ }^{19}$ where the temperature has been set to 0 . (Note that if one were to consider only moves that were acceptable rather than trying and then testing, one would have instead a random "neutral" walk performing "myopic" ant dynamics ${ }^{20}$.) In practice, we continue the random walk until the target structure is reached, or until a maximum number of trial mutations is reached.

In our work, we allow only neutral or improving moves in the landscape, which corresponds to zero temperature dynamics in physical systems, or hill climbing in optimisation theory. The trajectories are stochastic and are influenced both by the initial genotype chosen (e.g. an arbitrary RNA sequence), and by the target (an arbitrary secondary structure). We thus need to average over many trajectories, and consider the dependence of our results on these choices.

In a more general context, one can consider the evolution of a population in the fitness landscape. If $\mu$ is the mutation rate and $N$ the population size, then the effective number of genotypes in a population scales approximately linearly with $N \mu$. When $N \mu \ll 1$, the population remains essentially monomorphic, and this 
is the case we focus on in this work. If instead $N \mu$ were large, the evolutionary dynamics would occur in a polymorphic population with many different genotypes.

\subsection{Equilibrium sampling under stabilizing selection}

To test whether the out-of-equilibrium evolutionary dynamics generates atypical genotypes, one must define the "null hypothesis"; clearly we want to compare to random genotypes in the fitness landscape. This means that we need to sample uniformly the fitness landscape for each given distance to the target phenotype; this means we focus on the genotypes that have a given fitness. The algorithmic procedure to do so is to start with any genotype in the fitness landscape with the specified fitness and then produce a long random walk with importance sampling using the Metropolis Monte Carlo algorithm ${ }^{19}$. In our RNA neutral ensemble context, one accepts only the mutations that do not change the distance to the target; this is then identical to dynamics under a particular stabilizing selection. A long Monte Carlo then provides genotypes satisfying that constraint and their probability distribution is uniform.

From this sampling, we shall obtain equilibrium averages in this space. We will be particularly interested in the mean mutational robustness, where the mutational robustness of a genotype is defined as the fraction of the single-base mutations that do not change the fitness; this is the same thing as the number of neighbors of this genotypye that belong to the neutral ensemble divided by its total number of neighbors.

\section{Slow evolutionary dynamics}

\subsection{Typical trajectories have long stasis times}

Consider a typical evolutionary trajectory starting from a random initial genotype. At the beginning, there is a high frequency of advantageous mutations, so the phenotypic distance $d_{p}$ to the target structure initially decreases fast. But at long times, as first realized in ${ }^{5}$, the frequency of favorable mutations becomes small and long periods of "stasis" appear where the fitness remains constant $7,5,6,8$. This is illustrated for two typical evolutionary trajectories in Fig. 2. Successive plateaus in fitness are separated by small changes in the Hamming distance to the target: the steps decrease this distance by 1 or 2 units typically, rarely more than that. Also, the time of a stasis period typically increases as the distance to the target decreases: one can speak of diminishing returns for the effects of mutations in approaching the target.

\subsection{Anomalously slow dynamics}

To claim that the convergence to the target is particularly slow, it is appropriate to have a comparison benchmark. For this, consider the situation where directional 


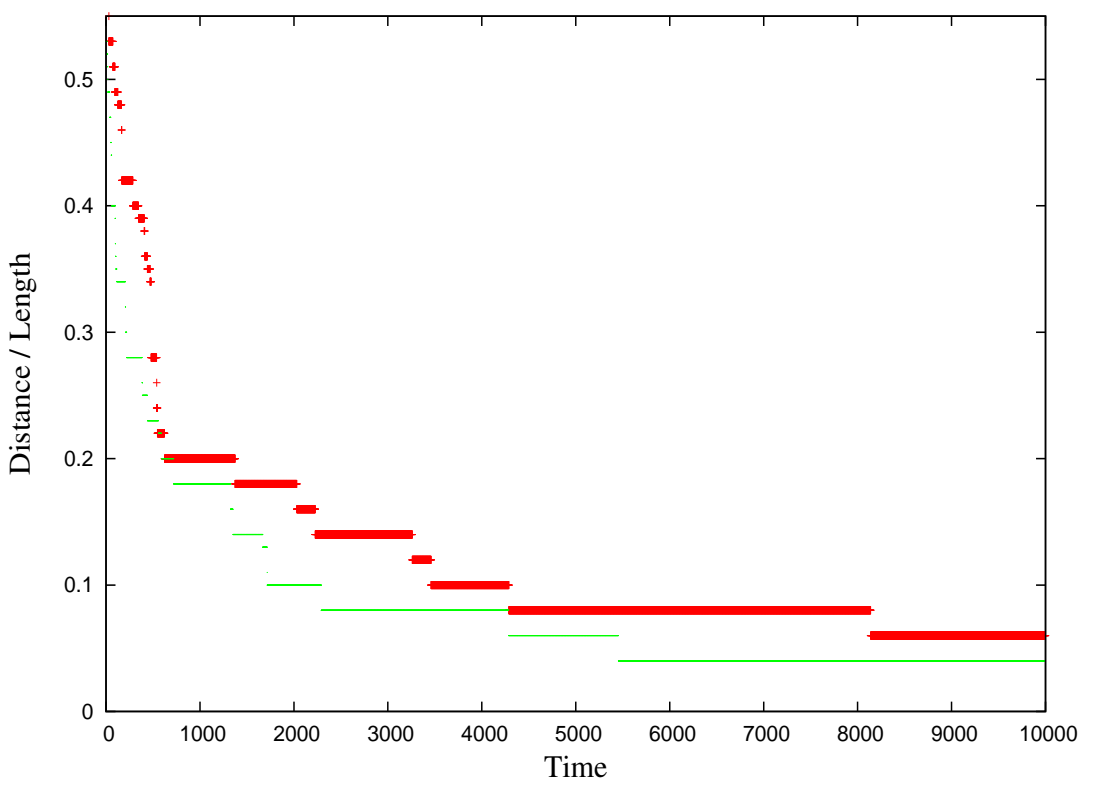

Fig. 2. Plot of the phenotypic distance to a target structure as a function of the number of mutation steps for chains of length 100. Shown are two typical trajectories, displaying periods of stasis.

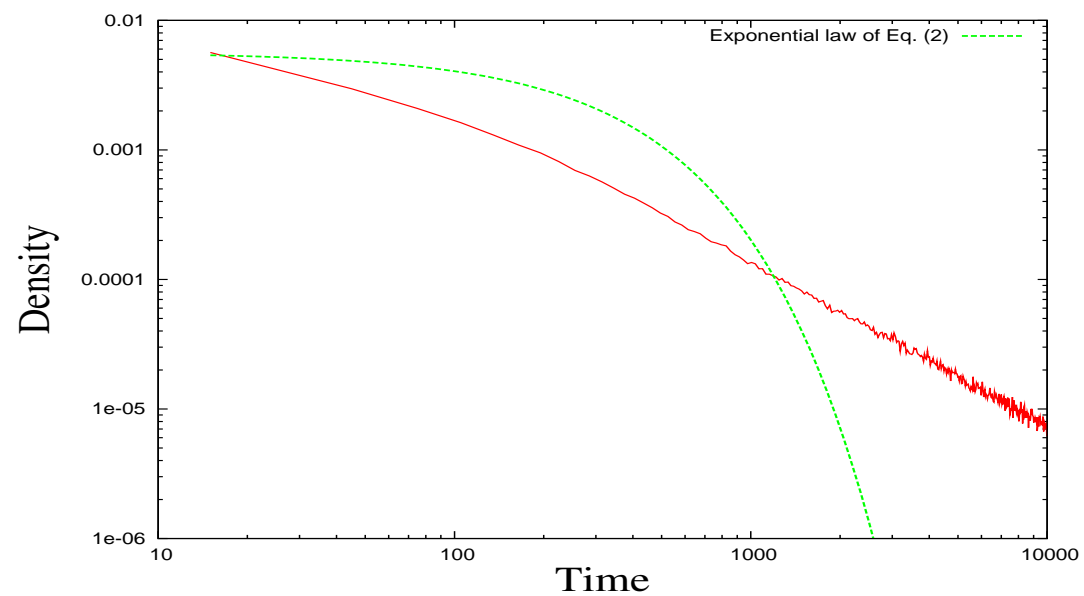

Fig. 3. Probability density for the times $\tau$ from the beginning of directional selection to transition points (separating two periods of stasis). ( $L=100$, averaged over 1000 trajectories for each of 30 randomly chosen targets.) Superposed is the analytical form following from Eq. 2.

selection is for a target genotype instead of a target phenotype. Just as for phenotypic distances, we define the genotypic distance of two genotypes $G_{1}$ and $G_{2}$ as 
$d_{G}=d_{H}\left(G_{1}, G_{2}\right) / L$ where $d_{H}\left(G_{1}, G_{2}\right)$ is the Hamming distance between the two strings defining the sequences for $G_{1}$ and $G_{2}$. If $d_{G}$ is then the distance between the current genotype and the target genotype, a mutation has a probability $d_{G} / 3$ to produce a strictly better genotype. It follows that the convergence to the target is exponential in time:

$$
\left\langle d_{G}(t)\right\rangle \approx d_{G}(0) e^{-t / 3 L} .
$$

Consider now the times $\tau$ when a favorable mutation arises; a transition between one stasis period and the next generates such an event which diminishes $d_{G}$ by $1 / L$. If $\rho(\tau)$ is the density of these times, we have

$$
-\frac{1}{L} \rho(\tau)=\left\langle d_{G}(\tau)-d_{G}(\tau-1)\right\rangle \approx \frac{d}{d \tau}\left\langle d_{G}(\tau)\right\rangle .
$$

We then obtain $\rho(\tau)=d_{G}(0) \exp (-\tau / 3 L) / 3$. This shows that selection for a target genotype leads to fast (exponential) relaxation.

The situation is completely different in our system where selection is instead for a target phenotype. Guided by the above analysis, we have determined the corresponding distribution $\rho(\tau)$ in this case. Fig. 3 shows the result when averaging over target and initial genotypes at $L=100$. This distribution has a very clear fat tail which seems compatible with a power law and not at all with the analytic form derived for selection according to genotypic distance. This same behavior is observed for the other values of $L$ tested (data not shown). One can thus say that convergence to the target under phenotypic selection is "slow", much slower than when compared to genotypic selection.

This difference between the selection types can also be seen by looking at the average distance to the target as a function of evolutionary time. In landscapes associated with disordered systems such as glasses or spin glasses, the relaxation processes encountered typically have non-exponential dynamics ${ }^{2}$. Empirically, two families of functions have been used to perform fits: the stretched exponential family which for our purposes corresponds to $\left\langle d_{P}\right\rangle \approx A \exp \left[-(t / T)^{\beta}\right]$, and the shifted power law family for which

$$
\left\langle d_{P}(t)\right\rangle \approx \frac{A}{(B+t)^{\nu}} .
$$

For small $L$, both types of fits lead to satisfactory results, but at larger $L$, the data favor a shifted power law behavior. In Fig. 4, we show that $\left\langle d_{P}(t)\right\rangle$ decreases rather slowly, roughly as an inverse power of time. This leads to a nearly straight line on a $\log -\log$ plot; also shown in that figure are the fits to shifted power laws. This overall behavior should be contrasted with the law (cf. Eq. 1) found when using selection for a target genotype: there the approach to the target was much faster.

\subsection{The slow dynamics is sensitive to the target}

To further analyse the slow approach towards a target structure, we investigated how the evolutionary dynamics changes with the target. First, we ran simulations 


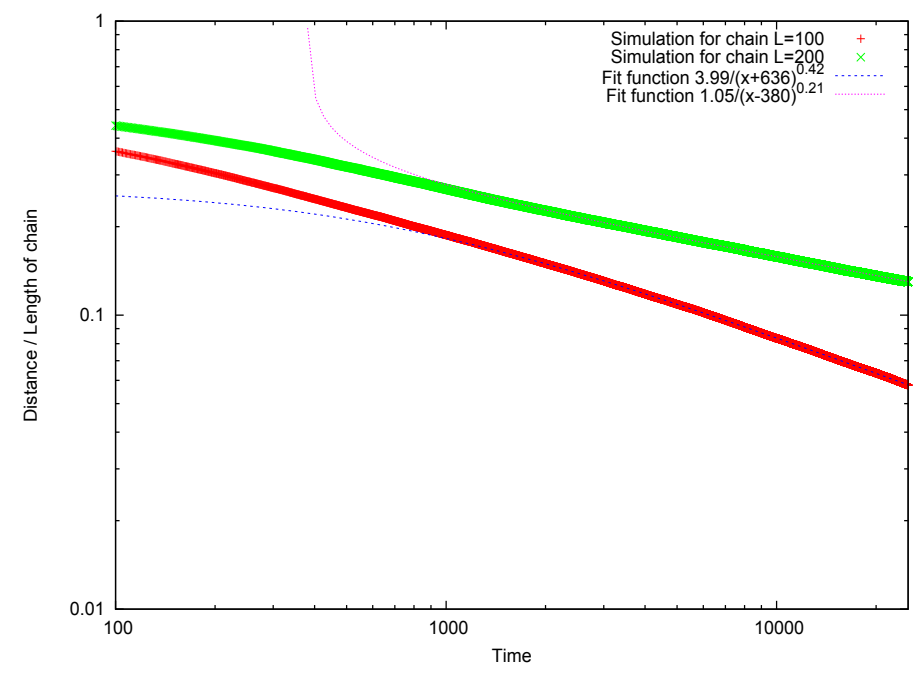

Fig. 4. Log-log plot of mean phenotypic distance to target structure as a function of the number of mutational steps for chains of length 100 and 200, when averaged over many initial genotypes and target structures. Also shown are the fits to a shifted power law (see text).

for genotypes of length 100 for different targets. In these simulations, for each target, we averaged the relaxation curves for different randomly generated initial genotypes. Fig. 5 illustrates how the relaxation is different for different target structures. We conclude that there is slow dynamics whose speed depends on the target structure, a conclusion that holds for all the values of the chain length $L$ we have investigated.

Although we cannot deal with arbitrary $L$ because of computational limitations, it is nevertheless relevant to ask whether this dependence on targets survives for arbitrarily large $L$. It seems possible that the relaxation behavior is not selfaveraging, i.e., that fluctuations associated with different targets do not become negligible when $L$ grows. To test this, we carried out simulations with multiple targets for different lengths of RNA chains, to determine whether the relaxation curves have smaller dispersion for the different targets when the chain length $L$ increases. We carried out simulations for thirty different targets, and averaged the relaxation curve for each target over 1000 evolutionary trajectories with random initial genotypes. We then measured the standard deviation $\sigma$ of the relative distance to the target phenotype at times where the mean distance (averaged over all curves) to the target was 10 percent of the chain length. Data are summarised in the following table. 


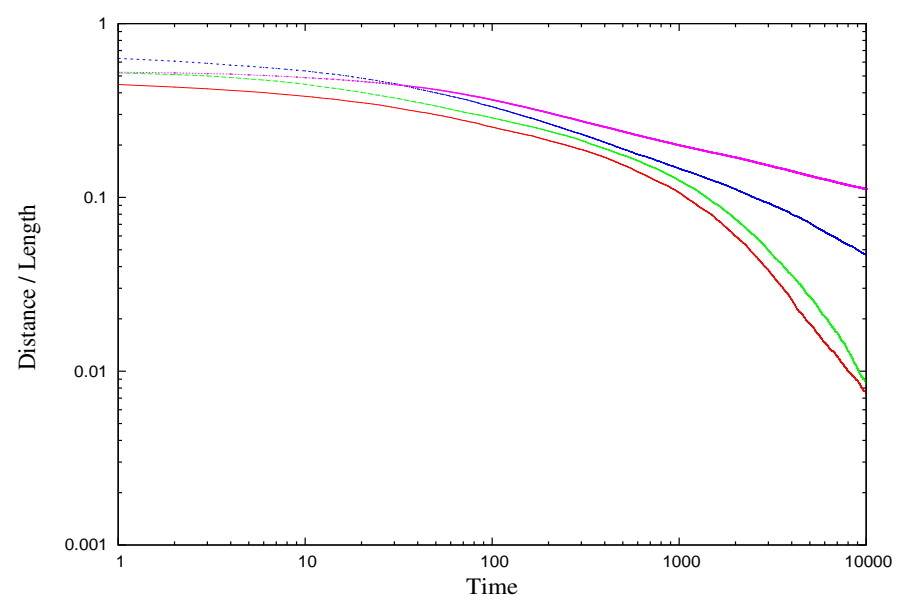

Fig. 5. Log-log plot of relative phenotypic distance from a target as a function of time for simulations with different targets (all of them of length 60). For each target, the plotted curve is an average over 1000 different trajectories.

\begin{tabular}{|l||l|l|}
\hline Chain length & $T_{0.1}$ & $\sigma$ \\
\hline 40 & 499 & 0.0346 \\
\hline 60 & 1439 & 0.02306 \\
\hline 80 & 3349 & 0.02475 \\
\hline 100 & 6933 & 0.02683 \\
\hline 120 & 8953 & 0.02626 \\
\hline
\end{tabular}

Here $T_{0.1}$ denotes the mean time at which the average distance to the target phenotype reaches the value $0.1 L, L$ being the chain length. The simulations were quite time-consuming which prevented us from testing lengths larger than 120. However, from the table we see that the dispersion $\sigma$ decreases initially, but then remains practically unchanged. The relaxation process towards different targets therefore is compatible with a non self-averaging behavior.

\section{Weak traces of evolutionary innovations}

The transition from one period of stasis to the next is due to an evolutionary "innovation". It is appropriate to ask whether the associated transition genotypes can be considered to be atypical according to some measurable quantity. Here we shall study the short term evolvability of the entry genotype in a period of stasis and compare it to that of random genotypes at the same distance to the target. For a given genotype we define its short-term evolvability ${ }^{23,24,25,26,12}$ as the fraction of single base mutations that lead to a phenotype with higher fitness. We found that the mean fraction of such beneficial mutations is approximately $40 \%$ lower for the entry genotype than for genotypes randomly sampled from the neutral ensemble. 


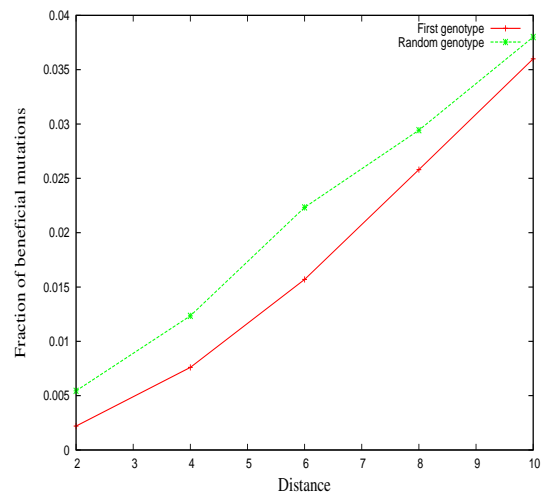

(a) Fraction of beneficial mutations.

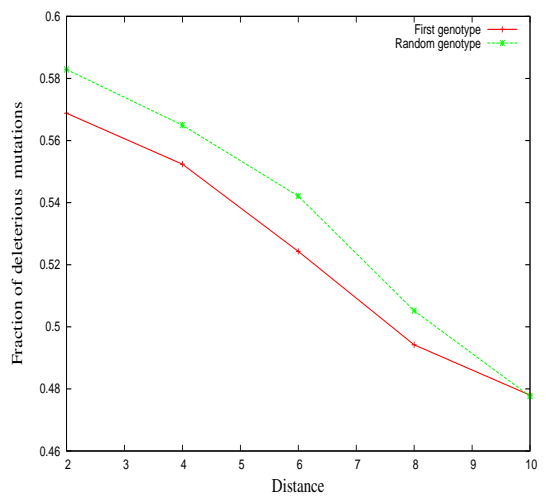

(b) Fraction of deleterious mutations.

Fig. 6. Comparison at $\mathrm{L}=40$ between entry ("first") genotypes and random (equilibrium) genotypes of the same fitness for the fraction of mutations that are beneficial (a) or deleterious (b). The $x$ axis gives the phenotypic distance to the target.

Thus with our definition, the entry genotype has an atypically low evolvability. How can this be qualitatively justified, at least naively? Suppose one thinks of the genotypes of given fitness as concentric spaces surrounding the target as russian dolls. Suppose further that these spaces have some "thickness". If our "entry" genotype is in the space $S\left(d_{P}\right)$, at phenotypic distance $d_{P}$ from the target, it will have fewer neighbors belonging to $S\left(d_{p}-1\right)$ than the typical genotype in $S\left(d_{P}\right)$.

However, we also find that the entry genotype has a smaller fraction of deleterious mutations than the neutral ensemble average. (These mutations produce a phenotype with increased distance to the target.) This result should be contrasted with what is naively expected. Indeed, by construction the entry genotype has one particular mutation which is known to be deleterious (taking it back to the previous plateau). Neglecting all other effects, one would predict that on average the entry genotype would have its fraction of deleterious mutations be $1 /(3 L)$ above that of genotypes in the neutral ensemble. Instead, the effect is four times larger and in the opposite direction.

During evolution on a plateau, one goes from the entry genotype (which we just saw is atypical according to some objective measure) to more random genotypes: to some extent, one loses the memory of the entry genotype through successive mutations. Is it a slow change of genotypes that is responsible for the stasis periods? To address this question, we monitored the distance between a mutating genotype and the entry genotype of the corresponding period of stasis. Because $L$ is moderately large in our simulations, the initial growth in distance is linear in the number of accepted mutations. At larger times, we see a saturation effect caused by multiple substitutions, as displayed in Fig. 7. Overall, the evolutionary dynamics on the neutral ensemble shows that even in the absence of phenotypic change, genotypes 


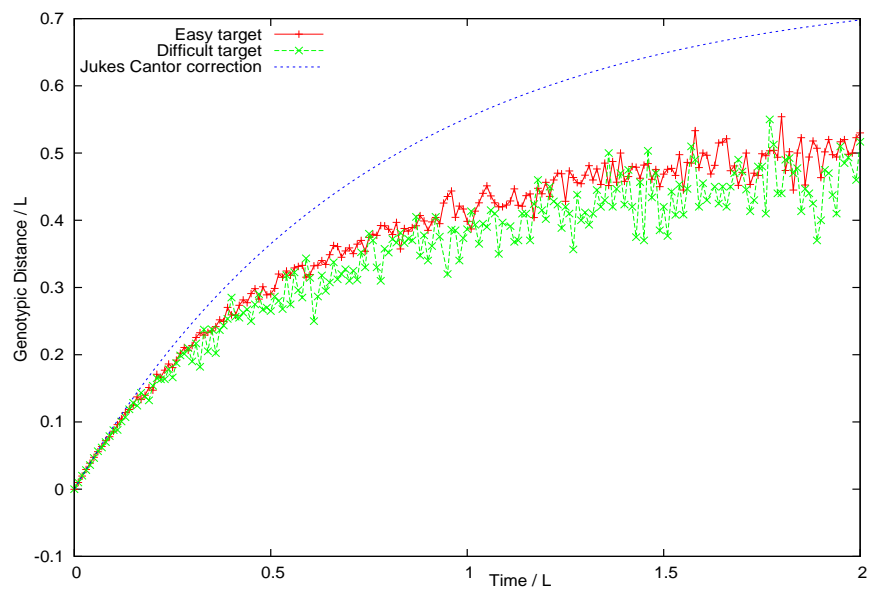

Fig. 7. Mean normalised genotypic distance to the genotype arising at the beginning of the current stasis period as a function of the number of accepted mutations divided by $L$, for chains of length $L=100$ (shown for two different targets). Also shown is the Jukes-Cantor correction for multiple substitutions, which is the function $f(x)=\frac{3}{4}\left(1-e^{-\frac{4}{3} x}\right)$ used in evolutionary biology to estimate the normalised distance from the initial genotype after $x$ mutations have taken place. The function $f(x)$ approximates the amount of change to be expected if evolutionary change is unconstrained. Our curves lie somewhat below $f(x)$ which is expected because genotype diffusion is constrained to remain on the neutral ensemble; nevertheless, the data show that diffusion in genotype space is rapid.

diffuse on a neutral ensemble at a rate not much slower than if their diffusion was not constrained by this set, until they discover a new phenotype closer to the target.

To understand the expected dynamics in the absence of selection consider that a given site mutates with probability $1 / L$ at each step, so the relaxation time scales as $L$. At long times one approaches the average distance $3 / 4 L$. This is the behavior, translated mathematically in the Jukes-Cantor formula ${ }^{22}$, that the uppermost curve in Fig.7 represents.

In summary, because of the intricate relation between genotype and phenotype, there can be slow dynamics in the approach to the target phenotype despite rapid evolutionary change of genotypes. In fact, as displayed in Fig. 7, the rate of change of genotypes does not seem to be significantly different when comparing easy and difficult targets for the directional selection.

\section{Discussion and conclusions}

Mappings from genotypes to phenotypes play a central role in biology, from the molecular scale up to whole organisms. Working at the level of RNA allowed us to use a framework for such mappings that is not biologically arbitrary, even though it is clearly idealised. One of this mapping's main advantages is that it is computationally tractable. Within this mapping, we showed a rich phenomenology of 
the evolutionary dynamics towards an optimum phenotype: (1) the "relaxation" towards the target undergoes severe slowing down as the target is approached; (2) this slowing down gives rise to stasis periods with fat tails, typical of what is expected in complex fitness landscapes; (3) the relaxation curves remain sensitive to the choice of the target, even in the limit of long RNA sequences; (4) the diffusion in genotype space during the periods of stasis is not slow, in obvious contrast with what happens at the level of phenotypes.

We observed that the probability of generating a favorable mutation decreases severely as one approaches the optimum, a property of the fitness landscape itself, i.e., the fraction beneficial mutations goes down in this limit, much faster than in systems undergoing exponential relaxation. Why this leads to inverse power laws remains open, just as in many other landscape problems coming from other fields. Furthermore, because the stasis periods are long, the few innovative genotypes appearing in an evolutionary trajectory represent a tiny fraction of the whole, and thus most genotypes visited have little trace of the out-of-equilibrium dynamics.

\section{References}

1. S. Gavrilets, Fitness Landscapes and the Origin of Species, Princeton University Press, 2004

2. A. P. Young, Spin Glasses and Random Fields, World Scientific Publishing, 1998

3. H. H. Hoos, T. Stützle, Stochastic Local Search: Foundations and Applications, Elsevier, 2005

4. N. Eldredge, J. N. Thompson, P. M. Brakefield, S. Gavrilets, D. Jablonski, J. B. C. Jackson, R. E. Lenski, B. S. Lieberman, M. A. McPeek,W. Miller III, The dynamics of evolutionary stasis, Paleobiology v. 31; no. 2_Suppl, June 2005

5. M. Huynen, P. Stadler W. Fontana: Smoothness within Ruggendess: The role of neutrality, Proc. Natl. Acad. Sci. USA, Vol. 93, 397-401 (1996).

6. W. Fontana, P. Schuster: Slow evolutionary dynamics of RNA structures and evolvability, Science 29, Vol. 280. no. 5368, pp. 1451 - 1455 (1998)

7. P. Schuster, W. Fontana, P. F. Stadler, I. L. Hofacker, From sequences to shapes and back: A case study in RNA secondary structures, Proc. R. Soc. Lond. B, 255, 279-284 (1994)

8. W. Fontana, P. Schuster, Shaping Space: the Possible and the Attainable in RNA Genotype-phenotype Mapping, J. Theor. Biology, Vol. 194, Issue 4, 491-515 (1998)

9. Gesteland, R.F., Atkins, J.F. (ed.): The RNA world, Cold Spring Harbor Laboratory Press, 1993

10. http://www.tbi.univie.ac.at/ ivo/RNA/

11. W. Fontana P. F. Stadler, E. G. Bornberg-Bauer, T. Griesmacher, I. L. Hofacker, M. Tacker, P. Tarazona, E. .D Weinberger,P. Schuster, RNA folding and combinatory landscapes, Phys. Rev. E 47, 1993

12. A. Wagner, Robustness and evolvability: a paradox resolved, Proc. Biol. Sci., Vol 275, 91-100, 2008

13. B. Voss, C. Meyer, R. Giegerich, Evaluating the predictability of conformational switching in RNA Bioinformatics 20: 1573-1582, 2004

14. P. F. Stadler, Landscapes and Their Correlation Functions J.Math.Chem. 20: 1-45, 1996 
15. C. Monthus, J.-P. Bouchaud, Models of traps and glass phenomenology, J. Phys. A: Gen., Vol. 29, 3847-3869, 1996

16. J.-P. Bouchaud, Weak ergodicity breaking and aging in disordered systems, J. Phys. I (France) Vol. 2, 1705-1713, 1992

17. B. D. Hughes, Random Walks and Random Environments, Oxford: Clarendon, 1996

18. S. Kauffman, S. Levin, Toward a general theory of adaptive walks on rugged landscapes, J. Theor. Biol. 1987, 128, 11-45

19. K. Binder, D. W. Heermann, Monte Carlo Simulation in Statistical Physics: An Introduction, Springer, 2002

20. E. Van Nimwegen, J. P. Crutchfield, M. Huynen, Neutral evolution of mutational robustness, Proc. Natl. Acad. Sci. USA, Vol. 96, 9716-9720, 1999

21. M. Zuker, P. Stiegler, Optimal computer folding of large RNA sequences using thermodynamics and auxiliary information, Nucleic Acids Res., Jan 10;9(1):133-48, 1981

22. M. Kimura, T. Ohta, On the stochastic model for estimation of mutational distance between homologous proteins, J. Mol. Evol., 87-90, 1972

23. M. A. Huynen, P. Stadler, W. Fontana, Smoothness within ruggedness: The role of neutrality in adaptation, Proc. Natl. Acad. Sci. USA, Vol. 93, 1996

24. M. Kirschner, J. Gerhart, Proc. Natl. Acad. Sci. USA, Evolvability, Vol. 95, 8420-8427, 1998

25. L. A. Meyers, F. D. Ancel, M. Lachmann, Evolution of Genetic Potential, PLoS Computational biology, Vol. 1, 2005

26. M. C. Cowperthwaite, L. A. Meyers, How Mutational Networks Shape Evolution: Lessons from RNA Models, Annu. Rev. Ecol. Evol. Syst. 38, 2007 